\title{
CONVEX REARRANGEMENTS, GENERALIZED LORENZ CURVES, AND CORRELATED GAUSSIAN DATA
}

\author{
YOURI DAVYDOV, DAVAR KHOSHNEVISAN, ZHAN SHI, AND RIČARDAS ZITIKIS
}

\begin{abstract}
We propose a statistical index for measuring the fluctuations of a stochastic process $\xi$. This index is based on the generalized Lorenz curves and (modified) Gini indices of econometric theory.

When $\xi$ is a fractional Brownian motion with Hurst index $\alpha \in(0,1)$, we develop a complete picture of the asymptotic theory of our index. In particular, we show that the asymptotic behavior of our proposed index depends critically on whether $\alpha \in\left(0, \frac{3}{4}\right), \alpha=\frac{3}{4}$, or $\alpha \in\left(\frac{3}{4}, 1\right)$. Furthermore, in the first two cases, there is a Gaussian limit law, while the third case has an explicit limit law that is in the second Wiener chaos.
\end{abstract}

\section{INTRODUCTION}

Suppose that $\xi:=\{\xi(x) ; x \geq 0\}$ is a given stochastic process and, to be concrete, we assume it starts at the origin; i.e., $\xi(0)=0$ almost surely. Suppose, in addition, that the process $\xi$ has been sampled at the end of $n$ consecutive time-intervals that we take to be $[0,1), \ldots,[n-1, n)$. In particular, the resulting sample is nothing but $\xi_{1}, \xi_{2}, \ldots, \xi_{n}$.

Next we let $X_{0}:=0$, and

$$
X_{i}:=\xi(i)-\xi(i-1), \quad i=1,2, \ldots, n .
$$

If we write the corresponding order statistics as $0=: X_{0 ; n} \leq X_{1: n} \leq \cdots \leq X_{n: n}$, then the convex rearrangement of the increments $\left\{X_{i}\right\}_{i=1}^{n}$ is the process $\mathrm{C}_{n}$ defined as follows:

$$
\mathrm{C}_{n}(x)=\sum_{i=0}^{[x]} X_{i: n}+(x-[x]) X_{[x]+1: n}, \quad{ }^{\forall} x \in[0, n] .
$$

A little thought shows that $\mathrm{C}_{n}$ is the piecewise-linear function that connects the points $\left\{\left(i, \sum_{j=1}^{i} X_{j: n}\right)\right\}_{i=0}^{n}$. This should be compared to the piecewise-linear interpolation of the points $\{\xi(i)\}_{i=1}^{n}$ which is obtained by connecting the points $\left\{\left(i, \sum_{j=1}^{i} X_{j}\right)\right\}_{i=0}^{n}$. Figure 1(a) shows an example of a process $\xi$ (shown as a thin line), and the piecewise-linear interpolation of $\{\xi(i)\}_{i=1}^{n}$ (shown as a thick line). Figure 1(b) contains the said interpolation of $\{\xi(i)\}_{i=1}^{n}$ (shown as a thin line), and the convex rearrangement process $\mathrm{C}_{n}$ (shown as a thick line).

Date: May 29, 2003.

1991 Mathematics Subject Classification. Primary. 60G18; Secondary. 60G15.

Key words and phrases. Convex rearrangements, Lorenz curves, Gini indices, fractional Brownian motion.

The work of Y. D. was partially supported by the RFBR Grant 99-01-00112.

The work of D. Kh. was partially supported by the NSF.

The work of R. Z. was partially supported by an NSERC of Canada individual research grant at the University of Western Ontario. 


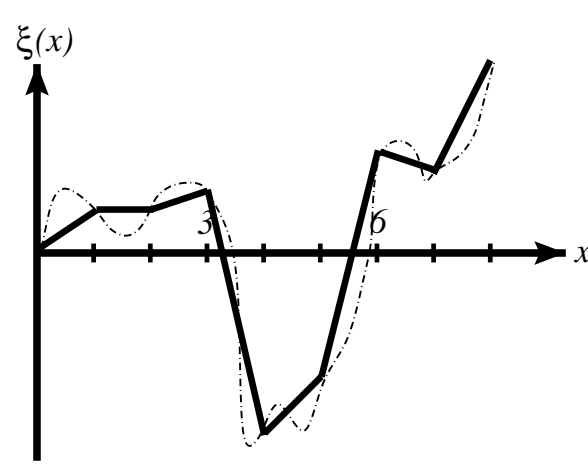

(a) The process $\xi$

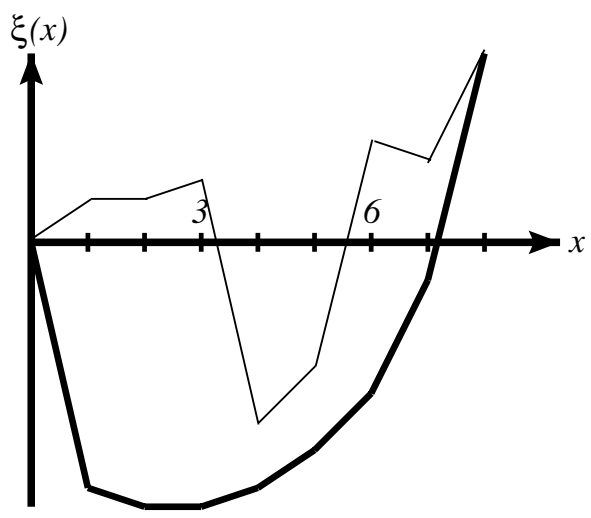

(b) The process $\mathrm{C}_{n}$

FiguRE 1. The convex rearrangement of $\xi$

The following are three of the significant features of the process $\mathrm{C}_{n}$ :

(1) For all $i=1, \ldots, n$, we have $\xi(i) \geq \mathrm{C}_{n}(i)$.

(2) $\xi$ and $\mathrm{C}_{n}$ start and end at the same values; i.e., $\xi(0)=\mathrm{C}_{n}(0)=0$ and $\xi(n)=\mathrm{C}_{n}(n)$.

$\left(3\right.$ The random function $\mathrm{C}_{n}$ is convex. In particular, $\mathrm{C}_{n}(x) \leq \frac{x}{n} \xi(n)=$ $\frac{x}{n} \mathrm{C}_{n}(n)$ for all $x \in[0, n]$.

Of course, $\mathrm{C}_{n}$ is convex because the slopes of the interpolated lines are increasing. (This explains why $\mathrm{C}_{n}$ is called the convex rearrangement of the data $\left\{X_{i}\right\}_{i=1}^{n}$.) However, there is a more abstract explanation for the said convexity that will be useful in our subsequent analysis. Note that

$$
\begin{aligned}
\mathrm{C}_{n}(x)=n \int_{0}^{x / n} F_{n}^{-1}(s) \mathrm{d} s & (x \in[0, n]), \text { where } \\
F_{n}(x)=\frac{1}{n} \sum_{j=1}^{n} \mathbf{1}_{\left\{X_{j} \leq x\right\}} & (x \in \mathbb{R})
\end{aligned}
$$

denotes the empirical distribution function of the increments $\left\{X_{i}\right\}_{i=1}^{n}$, and $F_{n}^{-1}(s):=$ $\inf \left\{x: F_{n}(x) \geq s\right\}$ is the corresponding quantile function. Thus, the convexity of $\mathrm{C}_{n}$ follows from taking two weak derivatives; cf. Remark A.2 below.

In view of the preceding remarks, we can now measure the fluctuations of the process $\xi$ over the time interval $[0, n]$ by calculating the area $A(n)$ that is covered between $\mathrm{C}_{n}$ and the line segment $x \mapsto \frac{x}{n} \xi(n)=\frac{x}{n} \mathrm{C}_{n}(n)$ over the time interval $[0, n]$. In other words,

$$
A(n):=\int_{0}^{1}\left(s \mathrm{C}_{n}(n)-\mathrm{C}_{n}(n s)\right) \mathrm{d} s=\int_{0}^{1}\left|\mathrm{C}_{n}(n s)-s \mathrm{C}_{n}(n)\right| \mathrm{d} s .
$$

Figure 2 depicts $A(n)$, for the process $\xi$ of Figure 1, as the area of the shaded region.

Next we describe a generalization of $A(n)$ that can be used to obtain a family of indices that measure the fluctuations of $\xi$. 


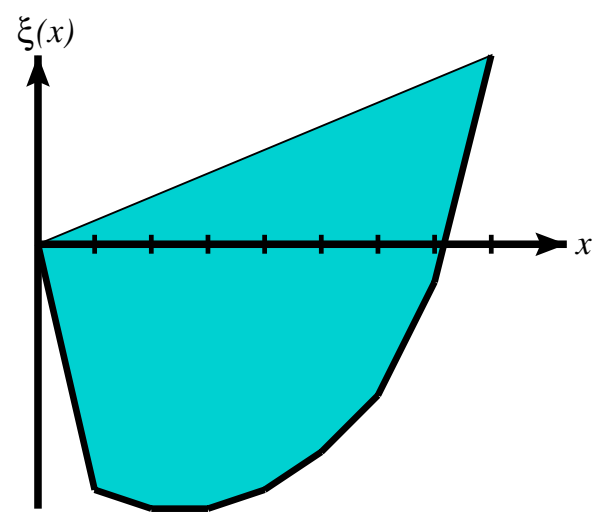

FIgURE 2. The index $A(n)$ is the area of the shaded region

Given a finite-mean distribution function $F$ on the line, we first recall the generalized Lorenz curve $\mathrm{GL}_{F}$, and define the mean-line function $\mu_{F}$, by

$$
\begin{array}{rlrl}
\mathrm{GL}_{F}(t) & :=\int_{0}^{t} F^{-1}(s) \mathrm{d} s, & \\
\mu_{F}(t) & :=t \mathrm{GL}_{F}(1)=t \int_{-\infty}^{\infty} x \mathrm{~d} F(x), & & \\
&
\end{array} \in[0,1],
$$

where $F^{-1}$ denotes the quantile function for $F$. The Lorenz curve, which is simply defined as $\mathrm{GL}_{F}(t) / \mathrm{GL}_{F}(1)$, was first introduced by Lorenz [Lor05], and the present general setting is attributed to Gastwirth [Gas71]. Then, for any fixed finite measure $\lambda$ on $[0,1]$, one can measure the fluctuations of $\xi$ by considering the index

$$
A_{p}(n):=\left\|\mathrm{GL}_{F_{n}}-\mu_{F_{n}}\right\|_{p} .
$$

As usual, $\|\cdots\|_{p}$ denotes the $L^{p}(\mathrm{~d} \lambda)$-norm; i.e.,

$$
\|f\|_{p}:=\left[\int_{0}^{1}|f(t)|^{p} \mathrm{~d} \lambda(t)\right]^{1 / p}, \quad{ }^{\forall} f \in L^{p}(\mathrm{~d} \lambda), p \in[1, \infty) .
$$

In particular, we note that $A(n)=A_{1}(n)$, where $\lambda$ denotes the Lebesgue measure on $[0,1]$.

Our index $A_{p}(n)$ is motivated by well-established concepts from econometric theory. For instance, the well-known Gini index of $\left\{X_{i}\right\}_{i=1}^{\infty}$, which is the most commonly-used measure of economic inequality, is none other than $2 A_{1}(n) / \bar{X}_{n}$, where $\bar{X}_{n}$ denotes the sample average of $X_{1}, \ldots, X_{n}$. This is indeed a measure of economic inequality, since it can be proved that

$$
\frac{2 A_{1}(n)}{\bar{X}_{n}}=\frac{1}{\bar{X}_{n}} \iint|x-y| \mathrm{d} F_{n}(x) \mathrm{d} F_{n}(y) .
$$

In words, the Gini index is the relative expected distance between two randomlyselected subsamples. For the proof of a more general fact that contains (1.8) see Mosler [Mos02, Proposition 7.1, and Remark 1 on p. 193].

The Gini index has played a central role in measuring economic inequality since the work of C. Gini in the early parts of the twentieth century; see David [Dav68] 
and Giorgi [Gio90, Gio93]. The more general index $A_{p}(n)$ is related to measures of economic inequality that involve weighing the underlying income population according to a finite measure $\mathrm{d} \lambda(t)=w(t) \mathrm{d} t$, and allows for the use of other $L^{p}$ norms. A more detailed discussion can be found in Barrett and Donald [BaD02] and Zitikis [Zit02, Zit03].

The main aim of this article is to develop some of the asymptotic theory of $A_{p}(n)$ in the case where $\xi$ is a Gaussian process with stationary increments and a nicely-behaved correlation function. ${ }^{1}$ From here, one can push further and use our results to construct asymptotic 95\%-confidence intervals (say) for variants of the Gini index of the population from which the $X_{i}$ 's have been drawn.

Although our methods can be used to study a large class of Gaussian processes, we restrict most of our attention to the case where $\xi$ is a fractional Brownian motion with parameter $\alpha \in(0,1)$. For such process, the main result of this paper implies the surprising fact that the asymptotic theory of our fluctuation indices goes through a phase transition at $\alpha=\frac{3}{4}$.

Recall that $\xi$ is a fractional Brownian motion of index $\alpha \in(0,1)$ if it is a continuous centered Gaussian process such that $\mathrm{E}\left\{|\xi(t)-\xi(s)|^{2}\right\}=|t-s|^{2 \alpha}$ for all $s, t \geq 0$. In this case, our asymptotics will imply that when $p \geq 2$ and $\lambda$ is the Lebesgue measure on $[0,1]$,

$$
A_{p}(n)-\frac{1}{(p+1)^{1 /(2 p) \sqrt{2 \pi}}}= \begin{cases}O_{\mathrm{P}}\left(n^{-1 / 2}\right) & \text { if } \alpha \in\left(0, \frac{3}{4}\right), \\ O_{\mathrm{P}}\left(n^{-1 / 2} \sqrt{\ln n}\right), & \text { if } \alpha=\frac{3}{4}, \\ O_{\mathrm{P}}\left(n^{-2(1-\alpha)}\right), & \text { if } \alpha \in\left(\frac{3}{4}, 1\right) .\end{cases}
$$

Moreover, after normalizing with the above rates, the left-hand side converges weakly to a normal law in the case that $\alpha \leq \frac{3}{4}$, and to an explicit law in the second Wiener chaos when $\alpha>\frac{3}{4}$.

We conclude this section with by noting that, in a rather different setting than ours, Guyon and Leon [GuL89] and Bardet [Bar99] have also noted different asymptotic behaviors with forms that depend on whether $\alpha<\frac{3}{4}$ or $\alpha>\frac{3}{4}$.

\section{The Main Result and First-Order Asymptotics}

Before we describe the main result of this paper, we need to introduce some notation.

Throughout, we use the standard notation for the normal distribution, viz.,

$$
\varphi(x):=\frac{e^{-x^{2} / 2}}{\sqrt{2 \pi}}, \quad \text { and } \quad \Phi(x)=\int_{-\infty}^{x} \varphi(y) \mathrm{d} y, \quad{ }^{\forall} x \in \mathbb{R} .
$$

Given any stationary Gaussian sequence $\left\{X_{i}\right\}_{i=1}^{\infty}$, we then write $\Phi_{n}$ for the corresponding empirical distribution function when the $X_{i}$ 's are standard normal; cf. (1.3) for the more general definition.

Throughout, $\mathcal{N}$ and $\boldsymbol{R}_{\alpha}$ denote independent random variables with the following respective distribution functions: $\mathcal{N}$ is standard normal; and $\boldsymbol{R}_{\alpha}$ is a Rosenblatt random variable [Ros61]. The Fourier-Laplace transform of $\mathcal{R}_{\alpha}$ is described by the

\footnotetext{
${ }^{1}$ We frequently assume that $\mathrm{E}\{\xi(t)\}=0$. This is not a great loss in generality, since many of the applications will then involve the process $\xi(t)+t \mu$ for a mean-constant $\mu$.
} 
following formula: For all $\alpha \in\left(\frac{3}{4}, 1\right)$, and $z \in \mathbb{C}$ with $|z|$ sufficiently small,

$$
\begin{aligned}
\mathrm{E}\left[e^{z \boldsymbol{\mathcal { R }}_{\alpha}}\right] & =\exp \left(\frac{1}{2} \sum_{k=2}^{\infty} \frac{(2 z)^{k}}{k} \int_{[0,1]^{k}}\left[\Psi_{k}(t)\right]^{-2(1-\alpha)} \mathrm{d} t\right), \text { where } \\
\Psi_{k}(t) & =\left|t_{2}-t_{1}\right| \times \cdots \times\left|t_{k}-t_{k-1}\right| \times\left|t_{1}-t_{k}\right|, \quad{ }^{\forall} t \in \mathbb{R}^{k} .
\end{aligned}
$$

Now we concentrate on the case where $\xi$ is fractional Brownian motion. The following is the main result of this paper.

Theorem 2.1 (Fractional Brownian Increments). Suppose $\xi$ is a fractional Brownian motion with Hurst parameter $\alpha \in(0,1)$. Then, as $n \rightarrow \infty$, the following hold:

(1) If $\alpha \in\left(0, \frac{3}{4}\right)$ and $p \geq 1$, then there exists a $\sigma:=\sigma(\alpha, p) \in(0, \infty)$ such that

$$
\sqrt{n}\left(A_{p}(n)-\left\|\varphi \circ \Phi^{-1}\right\|_{p}\right) \stackrel{d}{\longrightarrow} \sigma\left\|\varphi \circ \Phi^{-1}\right\|_{p}^{1-p} \mathcal{N}
$$

(2) If $\alpha=\frac{3}{4}$ and $p \geq 2$, then

$$
\sqrt{\frac{n}{\ln n}}\left(A_{p}(n)-\left\|\varphi \circ \Phi^{-1}\right\|_{p}\right) \stackrel{d}{\longrightarrow} \frac{9}{4 \sqrt{2}}\left\|\varphi \circ \Phi^{-1}\right\|_{p} \mathcal{N} .
$$

(3) If $\alpha \in\left(\frac{3}{4}, 1\right)$ and $p>2$, then

$$
n^{2(1-\alpha)}\left(A_{p}(n)-\left\|\varphi \circ \Phi^{-1}\right\|_{p}\right) \stackrel{d}{\longrightarrow} \frac{1}{2}\left\|\varphi \circ \Phi^{-1}\right\|_{p}\left(\mathcal{N}^{2}+3 \mathcal{R}_{\alpha}\right) .
$$

(4) If $\alpha \in\left[\frac{3}{4}, 1\right)$ and $p \in[1,2)$, then (2.4) and (2.5) both hold as long as there exists a $\theta>2-p$ such that

$$
\int_{0}^{1} s^{-\theta}(1-s)^{-\theta} \mathrm{d} \lambda(s)<+\infty
$$

Remark 2.2. In principle, $\sigma$ can be computed; cf. (2.17) and (3.3) below.

Remark 2.3. When $\lambda$ is the Lebesgue measure, then (2.6) holds for all $\theta \in(0,1)$, but not for $\theta=1$. In particular, the only case left uncovered by parts 2 and 3 of Theorem 2.1 is $p=1$. This leads us to the following:

Conjecture 2.3 ${ }^{\star}$. If $\alpha \in\left[\frac{3}{4}, 1\right)$ and $\lambda$ is the Lebesgue measure, then (2.4) and (2.5) continue to hold for $p=1$.

We now digress slightly by first studying a more general problem. To do so, we dispense with the process $\xi$ altogether, and consider a stationary sequence $\left\{X_{i}\right\}_{i=1}^{\infty}$ of integrable random variables whose common distribution is $F$. We will impose various conditions on $F$, and on the dependence structure of the $X_{i}$ 's, throughout.

Since $\left\|\mu_{F}-\mathrm{GL}_{F}\right\|_{p}$ is the "population" version of the index $A_{p}(n)$, it is natural to expect that, given a nice $F$ and for a nice dependence structure among the $X_{i}$ 's, $\lim _{n \rightarrow \infty} A_{p}(n)=\left\|\mu_{F}-\mathrm{GL}_{F}\right\|_{p}$. Therefore, we begin by making this statement precise.

Theorem 2.4. If $\left\{X_{i}\right\}_{i=1}^{\infty}$ is a strictly stationary and ergodic sequence, then almost surely, $\lim _{n \rightarrow \infty} A_{p}(n)=\left\|\mu_{F}-\mathrm{GL}_{F}\right\|_{p}$. 
Proof. We start with the following inequalities:

$$
\begin{aligned}
\left|A_{p}(n)-\left\|\mu_{F}-\mathrm{GL}_{F}\right\|_{p}\right| & \leq\left\|\left(\mu_{F_{n}}-\mathrm{GL}_{F_{n}}\right)-\left(\mu_{F}-\mathrm{GL}_{F}\right)\right\|_{p} \\
& \leq\left\|\mu_{F_{n}}-\mu_{F}\right\|_{p}+\left\|\mathrm{GL}_{F_{n}}-\mathrm{GL}_{F}\right\|_{p} \\
& \leq \lambda([0,1]) \cdot\left(\left|\bar{X}_{n}-\mu\right|+\left\|\mathrm{GL}_{F_{n}}-\mathrm{GL}_{F}\right\|_{\infty}\right),
\end{aligned}
$$

where $\|\cdot\|_{\infty}$ denotes the sup-norm here and throughout.

Thanks to the Birkhoff-Khintchine ergodic theorem, $\lim _{n} \bar{X}_{n \rightarrow \infty}=\mu$, a.s., and so it remains to prove that $\lim _{n \rightarrow \infty}\left\|\mathrm{GL}_{F_{n}}-\mathrm{GL}_{F}\right\|_{\infty}=0$, a.s. For this we use the inequality,

$$
\left\|\mathrm{GL}_{F_{n}}-\mathrm{GL}_{F}\right\|_{\infty} \leq d_{1}\left(F_{n}, F\right):=\int_{0}^{1}\left|F_{n}^{-1}(s)-F^{-1}(s)\right| \mathrm{d} s .
$$

The quantity $d_{1}\left(F_{n}, F\right)$ is the Dobrushin distance between $F_{n}$ and $F$; cf. [Dob70]. When the $X_{i}$ 's are independent, it is well known that $\lim _{n \rightarrow \infty} d_{1}\left(F_{n}, F\right)=0$ almost surely; see Shorack and Wellner [ShW86, Exercise 9, p. 66]. This fact continues to hold in the more general case of stationary and ergodic sequences; see Davydov and Zitikis [DaZ01].

Now, as a first step toward proving Theorem 2.1, we apply the preceding theorem to the increments of a fractional Brownian motion.

Corollary 2.5. Choose some $p \geq 1$, and consider $\left\{X_{i}\right\}_{i=1}^{\infty}$ to be the increments of a fractional Brownian motion $\xi$ with Hurst parameter $\alpha \in(0,1)$. Then the sample index of the fluctuations of $\xi$ converges to the theoretical one; i.e.,

$$
\lim _{n \rightarrow \infty} A_{p}(n)=\left\|\varphi \circ \Phi^{-1}\right\|_{p}, \quad \text { a.s. }
$$

In particular, when $\lambda$ is the Lebesgue measure on $[0,1]$,

$$
\lim _{n \rightarrow \infty} A_{p}(n)=\frac{1}{(p+1)^{1 /(2 p)} \sqrt{2 \pi}}, \quad \text { a.s. }
$$

Proof. The ergodicity of the $X_{i}$ 's was first proved in Maruyama [Mar49, Theorem 9(ii), p. 58]. [For a modern and elementary proof, see Rosenblatt [Ros61, p. 432].] Therefore, the two displays follow readily from Theorem 2.4, and Remarks A.1 and A.3 of the appendix.

Corollary 2.5 relied heavily on the ergodicity of $\left\{X_{i}\right\}_{i=1}^{\infty}$ which is equivalent to the convergence of $\operatorname{Cov}\left(X_{1}, X_{k}\right)$ to 0 in the sense of Cesàro. This, in turn, follows from $\lim _{k \rightarrow \infty} \operatorname{Cov}\left(X_{1}, X_{k}\right)=0$ whose verification is outlined in Remark A.4. Given these observations, it should not come as a surprise then that the rate of convergence in Corollary 2.5 depends on the rate of decay of the said correlation function.

Recall that a (weakly) stationary sequence $\left\{X_{i}\right\}_{i=1}^{\infty}$ is called short-range dependent if

$$
\sum_{k=1}^{\infty}\left|\operatorname{Cov}\left(X_{1}, X_{1+k}\right)\right|<\infty .
$$

Theorem 2.6. Let $p \geq 1$, and let $\left\{X_{i}\right\}_{i=1}^{\infty}$ be a short-range dependent and stationary Gaussian process with distribution $F$. Then there exists a nontrivial constant $\sigma:=\sigma(F ; \alpha, p)$ such that

$$
\sqrt{n}\left(A_{p}(n)-\left\|\mu_{F}-\mathrm{GL}_{F}\right\|_{p}\right) \stackrel{d}{\longrightarrow}\left\|\mu_{F}-\mathrm{GL}_{F}\right\|_{p}^{1-p} \sigma \mathcal{N} .
$$


Moreover, the following is one particular representation for $\sigma:=\sigma(F ; \alpha, p)$ :

$$
\sigma^{2}:=\operatorname{Var}(\Lambda(Z))
$$

where the linear functional $\Lambda$ is defined by the formula,

$$
\Lambda(Z):=\int_{0}^{1}\left|\mu_{F}(t)-\mathrm{GL}_{F}(t)\right|^{p-1} Z(t) \mathrm{d} \lambda(t),
$$

$Z(t):=Y(t)-t Y(1)$ is the Gaussian bridge corresponding to the process $Y(t):=$ $\int_{-\infty}^{F^{-1}(t)} \Gamma(x) \mathrm{d} x$, and $\Gamma$ is a centered Gaussian process with

$$
\operatorname{Cov}(\Gamma(x), \Gamma(y))
$$

$$
=F(x \wedge y)-F(x) F(y)+2 \sum_{k=1}^{\infty} \operatorname{Cov}\left(\mathbf{1}_{\left\{X_{1} \leq x\right\}}, \mathbf{1}_{\left\{X_{1+k} \leq y\right\}}\right) .
$$

Thanks to (2.11), the infinite series in (2.15) converges absolutely for any $x, y \in$ $\mathbb{R}$; cf. Lemma A.5 below.

Before proving Theorem 2.6, we use it to derive the first, and the simplest, part of Theorem 2.1.

Proof of Theorem 2.1: Part 1. If $\left\{X_{i}\right\}_{i=1}^{\infty}$ are the increments of an index- $\alpha$ fractional Brownian motion, then we use the fact that $-\mathrm{GL}_{\Phi}=\varphi \circ \Phi^{-1}$ (Remark A.3), and apply Theorem 2.6 with $F:=\Phi$ to deduce that

$$
\sqrt{n}\left(A_{p}(n)-\left\|\varphi \circ \Phi^{-1}\right\|_{p}\right) \stackrel{d}{\longrightarrow}\left\|\varphi \circ \Phi^{-1}\right\|_{p}^{1-p} \sigma \mathcal{N},
$$

where

$$
\sigma^{2}:=\operatorname{Var}\left(\int_{0}^{1}\left|\varphi \circ \Phi^{-1}(t)\right|^{p-1} Z(t) \mathrm{d} \lambda(t)\right) .
$$

Moreover, $Z(t):=Y(t)-t Y(1)$ is the Gaussian bridge corresponding to the process $Y(t):=\int_{-\infty}^{\Phi^{-1}(t)} \Gamma(x) \mathrm{d} x$, and $\Gamma$ is a centered Gaussian process with

$\operatorname{Cov}(\Gamma(x), \Gamma(y))$

$$
=\Phi(x \wedge y)-\Phi(x) \Phi(y)+2 \sum_{k=1}^{\infty} \operatorname{Cov}\left(\mathbf{1}_{\left\{X_{1} \leq x\right\}}, \mathbf{1}_{\left\{X_{1+k} \leq y\right\}}\right) .
$$

This completes the proof of the first portion of Theorem 2.1.

We are ready to present the following.

Proof of Theorem 2.6. We begin by noting that the condition $\alpha \in\left(0, \frac{1}{2}\right]$ is equivalent to the short-range dependence of $\left\{X_{i}\right\}_{i=1}^{\infty}$; cf. Remark A.4.

Define the functional $T(F):=\left\|\mu_{F}-\mathrm{GL}_{F}\right\|_{p}$, and note that $A_{p}(n)=T\left(F_{n}\right)$. Therefore, an applications of the Taylor formula reveals that

$$
\sqrt{n}\left|A_{p}(n)-\left\|\mu_{F}-\mathrm{GL}_{F}\right\|_{p}-\Lambda\left(Z_{n}\right)\right|=o_{\mathrm{P}}(1),
$$

where $\Lambda$ is the linear functional that was defined in Theorem 2.6, and the stochastic process $Z_{n}$ is defined by the formula,

$$
Z_{n}:=\left(\mu_{F_{n}}-\mathrm{GL}_{F_{n}}\right)-\left(\mu_{F}-\mathrm{GL}_{F}\right) .
$$

In the case of short-range dependent increments we have the following [DaZ01]:

$$
\sqrt{n}\left(\mathrm{GL}_{F_{n}}-\mathrm{GL}_{F}\right) \Longrightarrow-Y
$$


where the process $Y$ is defined in Theorem 2.6, and $\Rightarrow$ denotes weak convergence in $C([0,1])$. Thus, $\sqrt{n} Z_{n} \Longrightarrow Z$, for the very same process $Z$ that was defined in Theorem 2.6. Now a standard continuity argument shows that $\sqrt{n} \Lambda\left(Z_{n}\right)$ converges in distribution to $\Lambda(Z)$, whence (2.12). Thus, our task is completed as soon as we prove that $\sigma<+\infty$.

Now uniformly for all $t \in[0,1]$,

$$
0 \leq \mu_{F}(t)-\mathrm{GL}_{F}(t) \leq 2 \int_{-\infty}^{\infty}|x| \mathrm{d} F(x)<+\infty .
$$

Thus, a little computation on the side shows that $\sigma<+\infty$ if we could prove that $\operatorname{Var}(Y(t))$ is bounded in $t$. Since $Y$ is centered, and proceeding somewhat informally, we have

$$
\operatorname{Var}(Y(t))=\int_{-\infty}^{F^{-1}(t)} \int_{-\infty}^{F^{-1}(t)} \operatorname{Cov}(\Gamma(x), \Gamma(y)) \mathrm{d} x \mathrm{~d} y
$$

[To make this completely rigorous, first replace $\int_{-\infty}^{F^{-1}(t)}$ by $\int_{-N}^{F^{-1}(t)}$, and then let $N \rightarrow \infty$.] Next we recall that $\alpha \leq \frac{1}{2}$ means that the process $\left\{X_{i}\right\}_{i=1}^{\infty}$ is negatively correlated; cf. (A.5). Therefore, Slepian's inequality [Slep62] and (2.18) together imply that $\operatorname{Cov}(\Gamma(x), \Gamma(y)) \leq F(x \wedge y)[1-F(x \vee y)]$, which is integrable $[\mathrm{d} x \times \mathrm{d} y]$. This and (2.23) together show that $\operatorname{Var}(Y(t))$ is bounded in $t$, and this completes our proof.

Next consider the case where $\left\{X_{i}\right\}_{i=1}^{\infty}$ is long-range dependent; i.e., the case when (2.11) fails to hold. To be concrete, we assume that $X_{i}$ 's are the increments of a fractional Brownian motion. Thus, by (A.4), $\alpha \in\left(\frac{1}{2}, 1\right)$.

In this case, (2.21) has the following analogue [DaZ01]:

$$
\frac{\sqrt{n}}{d_{n}}\left(\mathrm{GL}_{\Phi_{n}}-\mathrm{GL}_{\Phi}\right) \Longrightarrow-Y^{*}
$$

where $Y^{*}(t):=t \mathcal{N}$, and $d_{n}$ is defined by the formula

$$
d_{n}^{2}:=1+2 \sum_{k=1}^{n-1}\left(1-\frac{k}{n}\right) \operatorname{Cov}\left(X_{1}, X_{1+k}\right) .
$$

Consequently, by a standard continuity argument,

$$
\frac{\sqrt{n}}{d_{n}}\left(A_{p}(n)-\left\|\varphi \circ \Phi^{-1}\right\|_{p}\right) \stackrel{d}{\longrightarrow}\left\|\varphi \circ \Phi^{-1}\right\|_{p}^{1-p} \Lambda\left(Z^{*}\right),
$$

where $Z^{*}(t):=Y^{*}(t)-t Y^{*}(1)=0$. Unfortunately, $\Lambda\left(Z^{*}\right) \equiv 0$, which means that, unlike the short-range dependent case, the long-range dependent case does not follow from general weak convergence principles. Of course, in the preceding display, $d_{n}=(c+o(1)) n^{2 \alpha-1}$ goes to infinity, so (2.26) is consistent with the remaining parts of Theorem 2.1. 


\section{Proof of Theorem 2.1: PARTs 2 And 3}

Consider the function,

$$
\begin{aligned}
G(u) & :=\int_{0}^{1}\left|\varphi \circ \Phi^{-1}(s)\right|^{p-1} g(u, s) \mathrm{d} \lambda(s), \text { where } \\
g(u, s) & :=\int_{-\infty}^{\Phi^{-1}(t)}\left[\mathbf{1}_{[u, \infty)}(x)-\Phi(x)\right] \mathrm{d} x-s \int_{-\infty}^{\infty}\left[\mathbf{1}_{[u, \infty)}(x)-\Phi(x)\right] \mathrm{d} x .
\end{aligned}
$$

Part 2 of Theorem 2.1 is contained within our next result.

Theorem 3.1. Let $p \geq 2$, and suppose $\left\{X_{i}\right\}_{i=1}^{\infty}$ are the increments of a fractional Brownian motion with index $\alpha \in\left(\frac{1}{2}, \frac{3}{4}\right)$. Then,

$$
\sqrt{n}\left(A_{p}(n)-\left\|\varphi \circ \Phi^{-1}\right\|_{p}\right) \stackrel{d}{\longrightarrow}\left\|\varphi \circ \Phi^{-1}\right\|_{p}^{1-p} \sigma \mathcal{N}
$$

where

$$
\sigma^{2}:=\sum_{k=1}^{\infty} \mathrm{E}\left[G\left(X_{1}\right) G\left(X_{1+k}\right)\right] \in(0, \infty)
$$

Before proving this, we first develop a series of technical lemmas.

Lemma 3.2. For all $\alpha \in(0,1)$ and $p>1$, there exists a constant $A:=A(\alpha, p) \in$ $(0, \infty)$ such that for all $u \in \mathbb{R},|G(u)| \leq A(1+|u|)$.

Proof. Write $\bar{F}:=1-F$ for any distribution function $F$, and note that

$$
\int_{-\infty}^{\infty}\left[\mathbf{1}_{[u, \infty)}(x)-\Phi(x)\right] \mathrm{d} x=\int_{u}^{\infty} \bar{\Phi}(x) \mathrm{d} x-\int_{-\infty}^{u} \Phi(x) \mathrm{d} x=-u .
$$

Next suppose that $u>\Phi^{-1}(s)$. Then, a similar argument shows that

$$
\int_{-\infty}^{\Phi^{-1}(s)}\left[\mathbf{1}_{[u, \infty)}(x)-\Phi(x)\right] \mathrm{d} x=-s \Phi^{-1}(s)-\varphi \circ \Phi^{-1}(s) .
$$

Finally, if $u \leq \Phi^{-1}(s)$, then

$$
\int_{-\infty}^{\Phi^{-1}(s)}\left[\mathbf{1}_{[u, \infty)}(x)-\Phi(x)\right] \mathrm{d} x=(1-s)\left(\Phi^{-1}(s)-u\right)+\varphi \circ \Phi^{-1}(u)-u s .
$$

By combining the preceding three displays we arrive at the neat formula:

$$
g(u, s)=s\left(u-\Phi^{-1}(s)\right)+\left(\Phi^{-1}(s)-u\right)^{+}+\varphi \circ \Phi^{-1}(s) .
$$

Thus, for all $u \in \mathbb{R}$ and $s \in[0,1],|g(u, s)| \leq|u|+\left|\Phi^{-1}(s)\right|+1$; i.e.,

$$
\sup _{s \in[0,1]}\left(\varphi \circ \Phi^{-1}(s)\right)^{p-1}|g(u, s)| \leq|u|+\sup _{x \in \mathbb{R}}|x| \varphi^{p-1}(x)+1 .
$$

The lemma follows upon integrating this $[\mathrm{d} \lambda]$.

Lemma 3.3. The infinite series in (3.3) converges absolutely if and only if $\alpha<\frac{3}{4}$.

In light of Giraitis and Sugrailis [GiS85, Lemma 5], this would follow immediately, once we prove that $G$ has Hermite rank 2. We dispense with this assertion next. 
Lemma 3.4. Suppose $H_{k}$ denotes the Hermite polynomial of rank $k$, and consider any $\alpha \in(0,1)$. Then, $G$ has Hermite rank 2 ; i.e., if we define $\left(G, H_{k}\right):=$ $\mathrm{E}\left\{G\left(X_{1}\right) H_{k}\left(X_{1}\right)\right\}$, then $\left(G, H_{0}\right)=\left(G, H_{1}\right)=0$, whereas $\left(G, H_{2}\right) \neq 0$. In addition,

$$
\left(G, H_{2}\right)=3\left\|\varphi \circ \Phi^{-1}\right\|_{p}^{p} .
$$

Proof. The assertion that $\left(G, H_{0}\right)$ is zero follows from the fact that $H_{0}(x) \equiv 1$.

Because $H_{1}(x)=x,\left(G, H_{1}\right)$ is zero if and only if $\mathrm{E}\left\{G\left(X_{1}\right) X_{1}\right\}$ is. This can be proved by showing that for all $s \in(0,1), \mathrm{E}\left\{g\left(X_{1}, s\right) X_{1}\right\}=0$. To prove this we appeal to (3.7) and write:

$$
\begin{aligned}
g(u, s) & =\omega_{0}(u, s)+\varphi \circ \Phi^{-1}(s), \text { where } \\
\omega_{0}(u, s) & :=s\left(u-\Phi^{-1}(s)\right)+\left(\Phi^{-1}(s)-u\right)^{+} .
\end{aligned}
$$

Clearly, $\mathrm{E}\left[g\left(X_{1}, s\right) X_{1}\right]=0$ if and only if $\mathrm{E}\left[\omega_{0}\left(X_{1}, s\right) X_{1}\right]=0$. But this is equivalent to checking that $\mathrm{E}\left[\left(\Phi^{-1}(s)-X_{1}\right)^{+} X_{1}\right]=-s$ which is easy to check directly.

In order to prove that $G$ has Hermite rank 2 we still need to verify that $\left(G, H_{2}\right) \neq$ 0 . Since $H_{2}(x)=x^{2}-1$, this amounts to proving $\mathrm{E}\left[G\left(X_{1}\right) X_{1}^{2}\right] \neq 0$. By $(3.10)$,

$$
\mathrm{E}\left[g\left(X_{1}, s\right) X_{1}^{2}\right]=\mathrm{E}\left[\omega_{0}\left(X_{1}, s\right) X_{1}^{2}\right]+\varphi \circ \Phi^{-1}(s) .
$$

By (3.10), and a few tedious calculations, $\mathrm{E}\left[\omega_{0}\left(X_{1}, s\right) X_{1}^{2}\right]=2 \varphi \circ \Phi^{-1}(s)$. Therefore, (3.11) implies that for all $s \in[0,1],\left(g(s, \cdot), H_{2}\right)=\mathrm{E}\left[g\left(X_{1}, s\right) X_{1}^{2}\right]=3 \varphi \circ \Phi^{-1}(s)>0$. The remainder of the lemma follows immediately from this.

Remark 3.5. We have actually proved the stronger result that for any $s \in(0,1)$, the Hermite rank of $g(\bullet, s)$ is 2 .

Lemma 3.6. If $\alpha<\frac{3}{4}$, and if $\sigma$ is defined by (3.3), then $\sigma>0$.

Proof. Since $\alpha<\frac{3}{4}$, Lemma 3.3 guarantees that we can apply the Wiener-Itô [Itô51] expansion of $G\left(X_{j}\right)$ in terms of the Hermite polynomials $\left\{H_{i}\right\}_{i=1}^{\infty}$. This yields the following: For all $m \geq 1$,

$$
G\left(X_{m}\right)=\sum_{i=0}^{\infty} \frac{1}{i !} H_{i}\left(X_{m}\right)\left(G, H_{i}\right)
$$

where the convergence takes place in $L^{2}(\mathrm{P})$. Because $G$ has Hermite rank 2, elementary properties of Hermite polynomials show that

$$
\mathrm{E}\left\{G\left(X_{1}\right) G\left(X_{1+k}\right)\right\}=\sum_{i=2}^{\infty} \frac{1}{i !}\left[\operatorname{Cov}\left(X_{1}, X_{1+k}\right)\right]^{i}\left(G, H_{i}\right)^{2} .
$$

In accord with (A.5), all the summands are nonnegative, and the first term is, in fact, strictly positive. This completes our proof.

Lemma 3.7. For all $\alpha \in\left(\frac{1}{2}, 1\right)$,

$$
\int_{0}^{1}\left|\Phi_{n}^{-1}(s)-\Phi^{-1}(s)\right| \mathrm{d} s=\int_{-\infty}^{\infty}\left|\Phi_{n}(x)-\Phi(x)\right| \mathrm{d} x=o_{\mathrm{P}}\left(n^{\alpha-1} \sqrt{\ln n}\right) .
$$

Remark 3.8. At the expense of writing a longer argument, one can, in fact, improve the rate to $O_{\mathrm{P}}\left(n^{\alpha-1}\right)$. The same improvement holds for Lemma 3.9 below. Also note that the above estimates the size of the Dobrushin distance between $\Phi_{n}$ and $\Phi$; cf. (2.8). 
Proof. In words, the first equality is stating that the area between $\Phi_{n}$ and $\Phi$ is the same as the area between their respective inverses. Thus, we need only to derive the stated little- $o$ estimate. With this aim in mind, define

$$
r_{n}(x):=\Phi_{n}(x)-\Phi(x)+\varphi(x) \bar{X}_{n} .
$$

Theorem 3.1 of Dehling and Taqqu [DeT89] and a Borel-Cantelli argument together prove that

$$
\left\|r_{n}\right\|_{\infty}=o\left(n^{\alpha-1}\right), \quad \text { a.s. }
$$

On the other hand, according to Taqqu [Taq75, Corollary 5.1],

$$
\bar{X}_{n}=O_{\mathrm{P}}\left(n^{\alpha-1}\right) \text {. }
$$

This readily yields the following:

$$
\int_{-2 \sqrt{\ln n}}^{2 \sqrt{\ln n}}\left|\Phi_{n}(x)-\Phi(x)\right| \mathrm{d} x=o_{\mathrm{P}}\left(n^{\alpha-1} \sqrt{\ln n}\right) .
$$

On the other hand, if we write $\bar{F}:=1-F$, then

$$
\mathrm{E}\left\{\int_{|x|>2 \sqrt{\ln n}}\left|\Phi_{n}(x)-\Phi(x)\right| \mathrm{d} x\right\} \leq 4 \mathrm{E}\left\{\int_{2 \sqrt{\ln n}}^{\infty} \bar{\Phi}(x) \mathrm{d} x\right\} .
$$

A direct computation reveals that for any $m>0, \int_{m}^{\infty} \bar{\Phi}(x) \mathrm{d} x \leq e^{-m^{2} / 2}$. Since $\alpha \in(0,1)$, this completes our proof.

Before stating our next lemma, let us define

$$
h_{n}:=\mu_{\Phi_{n}}-\left(\mathrm{GL}_{\Phi_{n}}-\mathrm{GL}_{\Phi}\right)=\mu_{\Phi_{n}}+\left(\varphi \circ \Phi^{-1}+\mathrm{GL}_{\Phi_{n}}\right) \text {. }
$$

Lemma 3.9. For all $\alpha \in\left(\frac{1}{2}, 1\right),\left\|h_{n}\right\|_{\infty}=o_{\mathrm{P}}\left(n^{\alpha-1} \sqrt{\ln n}\right)$.

Proof. Clearly, $\left\|h_{n}\right\|_{\infty} \leq\left|\bar{X}_{n}\right|+\left\|\mathrm{GL}_{\Phi_{n}}-\mathrm{GL}_{\Phi}\right\|_{\infty}$. Since $\left\|\mathrm{GL}_{\Phi_{n}}-\mathrm{GL}_{\Phi}\right\|_{\infty}$ does not exceed $\int_{-\infty}^{\infty}\left|\Phi_{n}(x)-\Phi(x)\right| \mathrm{d} x$, Lemma 3.7 and (3.17) together finish the proof.

Consider the processes $\beta^{U}$ and $\gamma$ defined below.

$$
\begin{aligned}
\beta_{n}^{U}(s) & :=\Phi_{n} \circ \Phi^{-1}(s)-s, \\
\gamma_{n}(s) & :=\Phi_{n}^{-1}(s)-\Phi^{-1}(s) .
\end{aligned}
$$

According to [DaZ01, Lemma 6.3], for any $\rho>0$, we have the statement $\sup _{s \in(0,1)} s^{\rho}(1-s)^{\rho}\left|\gamma_{n}(s)\right|=o_{\mathrm{P}}(1)$. A little computation on the side shows that this is equivalent to the statement that for all $\rho>0$,

$$
\left\|\left(\varphi \circ \Phi^{-1}\right)^{\rho} \gamma_{n}\right\|_{\infty}=o_{\mathrm{P}}(1)
$$

Next, we estimate the supremum norm of $\beta^{U}$. Moreover, we use (3.22) to improve itself in the case that $\rho>1$.

Proposition 3.10. If $\alpha \in\left(\frac{1}{2}, 1\right)$, then $\left\|\beta_{n}^{U}\right\|_{\infty}=O_{\mathrm{P}}\left(n^{\alpha-1}\right)$. In addition, for all $\rho>1,\left\|\left(\varphi \circ \Phi^{-1}\right)^{\rho} \gamma_{n}\right\|_{\infty}=O_{\mathrm{P}}\left(n^{\alpha-1}\right)$.

Proof. Recall $r_{n}$ from (3.15) and note that $\beta_{n}^{U}(s)=r_{n} \circ \Phi^{-1}(s)-\varphi \circ \Phi^{-1}(s) \bar{X}_{n}$. Therefore, by (3.16) and (3.17), $\left\|\beta_{n}^{U}\right\|_{\infty}=O_{\mathrm{P}}\left(n^{\alpha-1}\right)$, as claimed. Next we derive the asserted estimate on $\gamma_{n}$.

By (3.15), (3.16), for any $x, y \in \mathbb{R}$, with probability one,

$$
\left[\Phi_{n}(x)-\Phi_{n}(y)\right]-[\Phi(x)-\Phi(y)]+\bar{X}_{n}[\varphi(x)-\varphi(y)]=o\left(n^{\alpha-1}\right) .
$$


Here, the little- $o$ term does not depend on the choice of $x$ and $y$. Since $\sup _{s \in(0,1)} \mid \Phi_{n}$ 。 $\Phi_{n}^{-1}(s)-s \mid \leq \frac{1}{n}$, we apply the preceding with $x:=\Phi^{-1}(s)$ and $y:=\Phi_{n}^{-1}(s)$, and obtain that uniformly in $s \in(0,1), \beta_{n}^{U}(s)+\int_{\Phi_{n}^{-1}(s)}^{\Phi_{n}^{-1}(s)} \varphi(x) \mathrm{d} x=O_{\mathrm{P}}\left(n^{\alpha-1}\right)$. Since $\left\|\beta_{n}^{U}\right\|_{\infty}=O_{\mathrm{P}}\left(n^{\alpha-1}\right)$, this leads to

$$
\sup _{s \in(0,1)}\left|\left(\varphi \circ \Phi^{-1}(s)\right)^{\rho} \int_{\Phi^{-1}(s)}^{\Phi_{n}^{-1}(s)} \varphi(x) \mathrm{d} x\right|=O_{\mathrm{P}}\left(n^{\alpha-1}\right) .
$$

By the Fubini-Tonelli theorem, for every $s \in(0,1)$,

$$
\begin{aligned}
\left|\int_{\Phi^{-1}(s)}^{\Phi_{n}^{-1}(s)} \varphi(x) \mathrm{d} x-\varphi \circ \Phi^{-1}(s) \gamma_{n}(s)\right| & =\left|\int_{\Phi^{-1}(s)}^{\Phi_{n}^{-1}(s)} \int_{\Phi^{-1}(s)}^{x} \varphi^{\prime}(y) \mathrm{d} y \mathrm{~d} x\right| \\
& \leq \frac{1}{2}\left\|\varphi^{\prime}\right\|_{\infty}\left|\gamma_{n}(s)\right|^{2} .
\end{aligned}
$$

As a result,

$$
\left(\varphi \circ \Phi^{-1}(s)\right)^{\rho}\left|\gamma_{n}(s)\right| \leq \frac{1}{2}\left\|\varphi^{\prime}\right\|_{\infty}\left(\varphi \circ \Phi^{-1}(s)\right)^{\rho-1}\left|\gamma_{n}(s)\right|^{2}+O_{\mathrm{P}}\left(n^{\alpha-1}\right) .
$$

According to (3.22), if $\varepsilon \in(0, \rho-1)$, then, $\left|\gamma_{n}(s)\right|^{2}=o_{\mathrm{P}}\left(\left(\varphi \circ \Phi^{-1}(s)\right)^{-\varepsilon}\right)$, uniformly for all $s \in(0,1)$. Therefore, given any fixed sequence $\chi_{n} \downarrow 0$,

$$
\sup _{s \in\left[\chi_{n}, 1-\chi_{n}\right]}\left|\left(\varphi \circ \Phi^{-1}(s)\right)^{\rho} \gamma_{n}(s)\right|=o_{\mathrm{P}}\left(\left(\varphi \circ \Phi^{-1}\left(\chi_{n}\right)\right)^{\rho-1-\varepsilon}\right)+O_{\mathrm{P}}\left(n^{\alpha-1}\right) .
$$

On the other hand, according to (3.22), for any $\varepsilon \in(0, \rho-1)$,

$$
\sup _{s \in(0,1) \backslash\left[\chi_{n}, 1-\chi_{n}\right]}\left(\varphi \circ \Phi^{-1}(s)\right)^{\rho}\left|\gamma_{n}(s)\right|=o_{\mathrm{P}}\left(\left(\varphi \circ \Phi^{-1}\left(\chi_{n}\right)\right)^{\rho-\varepsilon}\right) .
$$

Combining the preceding displays leads to the following: For all $\varepsilon \in(0, \rho-1)$,

$$
\sup _{s \in(0,1)}\left|\left(\varphi \circ \Phi^{-1}(s)\right)^{\rho} \gamma_{n}(s)\right|=o_{\mathrm{P}}\left(\left(\varphi \circ \Phi^{-1}\left(\chi_{n}\right)\right)^{\rho-1-\varepsilon}\right)+O_{\mathrm{P}}\left(n^{\alpha-1}\right) .
$$

The proposition follows upon letting $\chi_{n}$ tend to 0 sufficiently rapidly.

Next, we recall the Vervaat process:

$$
V_{n}(s):=\int_{0}^{s}\left[\Phi_{n}^{-1}(u)-\Phi^{-1}(u)\right] \mathrm{d} u+\int_{-\infty}^{\Phi^{-1}(s)}\left[\Phi_{n}(x)-\Phi(x)\right] \mathrm{d} x .
$$

Lemma 3.11. For all $\alpha \in\left(\frac{1}{2}, 1\right)$ and $\rho>1,\left\|\left(\varphi \circ \Phi^{-1}\right)^{\rho} V_{n}\right\|_{\infty}=O_{\mathrm{P}}\left(n^{2 \alpha-2}\right)$.

Proof. It is well known that $0 \leq V_{n}(s) \leq\left|\beta_{n}^{U}(s) \gamma_{n}(s)\right|$, where $\gamma_{n}$ and $\beta_{n}^{U}$ are defined in (3.21). This is a geometric inequality that is explained, for example, in Zitikis [Zit98, eq.'s 1.9 and 1.12]. The lemma follows from the said inequality and Proposition 3.10.

We are now in a position to prove Theorem 3.1.

Proof of Theorem 3.1. Let

$$
\zeta(\tau):=\left\|-\mathrm{GL}_{\Phi}+\tau h_{n}\right\|_{p}=\left\|\varphi \circ \Phi^{-1}+\tau h_{n}\right\|_{p} .
$$

By the Taylor formula,

$$
A_{p}(n)-\left\|\varphi \circ \Phi^{-1}\right\|_{p}=\zeta^{\prime}(0)+\int_{0}^{1}\left[\zeta^{\prime}(\tau)-\zeta^{\prime}(0)\right] \mathrm{d} \tau .
$$


One can compute directly to see that the preceding terms are described as follows:

$$
\zeta^{\prime}(\tau)=[\zeta(\tau)]^{1-p} \int_{0}^{1}\left[\varphi \circ \Phi^{-1}(s)+\tau h_{n}(s)\right]^{p-1} h_{n}(s) \mathrm{d} \lambda(s) .
$$

In particular, the main term $\zeta^{\prime}(0)$ of $(3.32)$ is

$$
\zeta^{\prime}(0)=\left\|\varphi \circ \Phi^{-1}\right\|_{p}^{1-p} \int_{0}^{1}\left[\varphi \circ \Phi^{-1}(s)\right]^{p-1} h_{n}(s) \mathrm{d} \lambda(s) .
$$

Recall $h_{n}$ and $V_{n}$, respectively from (3.20) and (3.30), and note that $h_{n}$ is the difference between the following two functions:

$$
\begin{gathered}
\mu_{\Phi_{n}}(s)=-\frac{s}{n} \sum_{i=1}^{n} \int_{-\infty}^{\infty}\left[\mathbf{1}_{\left\{X_{i} \leq x\right\}}-\Phi(x)\right] \mathrm{d} x \\
\operatorname{GL}_{\Phi_{n}}(s)-\mathrm{GL}_{\Phi}(s)=-\frac{1}{n} \sum_{i=1}^{n} \int_{-\infty}^{\Phi^{-1}(s)}\left[\mathbf{1}_{\left\{X_{i} \leq x\right\}}-\Phi(x)\right] \mathrm{d} x+V_{n}(s) .
\end{gathered}
$$

By (3.1) and (3.35),

$$
\begin{aligned}
\zeta^{\prime}(0) & =\left\|\varphi \circ \Phi^{-1}\right\|_{p}^{1-p}\left(\frac{1}{n} \sum_{i=1}^{n} G\left(X_{i}\right)-\int_{0}^{1}\left[\varphi \circ \Phi^{-1}(s)\right]^{p-1} V_{n}(s) \mathrm{d} \lambda(s)\right) \\
& =\frac{\left\|\varphi \circ \Phi^{-1}\right\|_{p}^{1-p}}{n} \sum_{i=1}^{n} G\left(X_{i}\right)+O_{\mathrm{P}}\left(n^{2 \alpha-2}\right) .
\end{aligned}
$$

The function $G$ is defined in (3.1), and the second equality follows from Lemma 3.11 [applied with $\rho:=p-1$ ]. On the other hand, according to Giraitis and Surgailis [GiS85, Theorem 5], and owing to Lemma 3.4,

$$
n^{-1 / 2} \sum_{i=1}^{n} G\left(X_{i}\right) \stackrel{d}{\longrightarrow} \sigma \mathcal{N} .
$$

Because $\alpha \in\left(\frac{1}{2}, \frac{3}{4}\right),(3.37),(3.36)$, and (3.32) together complete our proof, provided that we prove the following: For all $\alpha \in\left(\frac{1}{2}, 1\right)$,

$$
\left\|\zeta^{\prime \prime}\right\|_{\infty}=O_{\mathrm{P}}\left(n^{2 \alpha-2}\right) \text {. }
$$

On the other hand, a direct computation reveals that

$$
\begin{aligned}
\zeta^{\prime \prime}(\tau)= & (1-p)[\zeta(\tau)]^{1-2 p}\left(\int_{0}^{1}\left(\varphi \circ \Phi^{-1}(s)+\tau h_{n}(s)\right)^{p-1} h_{n}(s) \mathrm{d} \lambda(s)\right)^{2} \\
& +(p-1)[\zeta(\tau)]^{1-p} \int_{0}^{1}\left(\varphi \circ \Phi^{-1}(s)+\tau h_{n}(s)\right)^{p-2} h_{n}^{2}(s) \mathrm{d} \lambda(s) .
\end{aligned}
$$

Since $\zeta$ and $\varphi \circ \Phi^{-1}$ are uniformly bounded, Lemma 3.9 proves (3.38), and hence Theorem 3.1 .

Proof of Theorem 2.1: Part 3. We follow closely the proof of the second portion of Theorem 2.6, all the time noting that (3.36) and (3.38) continue to hold in the 
present setting. On the other hand, when $\alpha=\frac{3}{4},(3.37)$ is replaced by the following:

$$
\begin{aligned}
& \left(n \sigma_{n}^{2}\right)^{-1 / 2} \sum_{i=1}^{n} G\left(X_{i}\right) \stackrel{d}{\longrightarrow} \mathcal{N}, \text { where } \\
& \sigma_{n}^{2}:=\sum_{k=1}^{n} \mathrm{E}\left[G\left(X_{1}\right) G\left(X_{1+k}\right)\right] .
\end{aligned}
$$

See [GiS85, Theorem 6]. Thus, in order to conclude, it suffices to estimate $\sigma_{n}$.

Recall that $G$ has Hermite rank 2. Thus, according to Itô [Itô51],

$$
\mathrm{E}\left[G\left(X_{1}\right) G\left(X_{1+k}\right)\right]=\sum_{j=2}^{\infty} \frac{1}{j !}\left[\operatorname{Cov}\left(X_{1}, X_{1+k}\right)\right]^{j}\left(\mathrm{E}\left[G\left(X_{1}\right) H_{j}\left(X_{1}\right)\right]\right)^{2} .
$$

Thanks to (A.3), $\operatorname{Cov}\left(X_{1}, X_{1+k}\right)=\left(\frac{3}{4}+o(1)\right) k^{-1 / 2}(k \rightarrow \infty)$. Therefore, as $k \rightarrow \infty$,

$$
\begin{aligned}
\mathrm{E}\left[G\left(X_{1}\right) G\left(X_{1+k}\right)\right] & =\sum_{j=2}^{\infty} \frac{1}{k^{j / 2} j !}\left(\frac{3}{4}+o(1)\right)^{j}\left(\mathrm{E}\left[G\left(X_{1}\right) H_{j}\left(X_{1}\right)\right]\right)^{2} \\
& =\left(\frac{9}{32}+o(1)\right) k^{-1} \times\left(\mathrm{E}\left[G\left(X_{1}\right) H_{2}\left(X_{1}\right)\right]\right)^{2} \\
& =\left(\frac{81}{32}+o(1)\right) k^{-1} \times\left\|\varphi \circ \Phi^{-1}\right\|_{p}^{2 p} .
\end{aligned}
$$

The second equality in (3.42) follows from $\mathrm{E}\left[G\left(X_{1}\right)^{2}\right]<\infty$ and $\mathrm{E}\left[H_{j}\left(X_{1}\right)^{2}\right]=j$ !; see (3.9) for the third equality. We have therefore proved that

$$
\sigma_{n}^{2}=\frac{81+o(1)}{32}\left\|\varphi \circ \Phi^{-1}\right\|_{p}^{2 p} \ln (n), \quad(n \rightarrow \infty) .
$$

Part 3 of Theorem 2.1 follows from this and (3.40).

\section{Proof of Theorem 2.1: Parts 3 And 4}

To a degree, our proof of the remaining portions of Theorem 2.1 follows the ideas developed in the proofs of its earlier parts. However, parts 3 and 4 are the more challenging portions of Theorem 2.1, since additional difficulties crop up. This is why the limit law is nonnormal, in fact.

Unless explicitly stated otherwise, we continue to use our earlier notation, but now we are assuming that $\alpha \in\left(\frac{3}{4}, 1\right)$.

Theorem 4.1. Under the assumptions of part 4 of Theorem 2.1,

$$
\begin{aligned}
& n^{2(1-\alpha)}\left(A_{p}(n)-\left\|\varphi \circ \Phi^{-1}\right\|_{p}\right) \\
& \quad=\frac{1}{2}\left\|\varphi \circ \Phi^{-1}\right\|_{p}^{p}\left\{\left(\frac{1}{n^{\alpha}} \sum_{i=1}^{n} X_{i}\right)^{2}+\frac{3}{n^{2 \alpha-1}} \sum_{i=1}^{n}\left(X_{i}^{2}-1\right)\right\}+o_{\mathrm{P}}(1) .
\end{aligned}
$$

Proof. We follow the proof of Theorem 3.1 until we reach (3.36). Since $\alpha \in\left(\frac{3}{4}, 1\right)$, it follows that the two terms $n^{-1} \sum_{i=1}^{n} G\left(X_{i}\right)$ and $\int_{0}^{1}\left|\varphi \circ \Phi^{-1}(s)\right|^{p-1} V_{n}(s) \mathrm{d} \lambda(s)$ are now asymptotically equivalent. [This contrasts with the proof when $\alpha<\frac{3}{4}$. In that case, we showed that $n^{-1} \sum_{i=1}^{n} G\left(X_{i}\right)$ is uniquely the dominant term.]

Our goal then is to estimate $n^{-1} \sum_{i=1}^{n} G\left(X_{i}\right)$ and $\int_{0}^{1}\left(\varphi \circ \Phi^{-1}(s)\right)^{p-1} V_{n}(s) \mathrm{d} \lambda(s)$ with objects that are a good deal simpler. We start with the term $n^{-1} \sum_{i=1}^{n} G\left(X_{i}\right)$. 
Recall that $G$ has Hermite rank 2 (Lemma 3.4), as well as finite absolute moments of all orders (Lemma 3.2). Because, in addition, $\alpha \in\left(\frac{3}{4}, 1\right)$, the Strong Reduction Theorem of Taqqu [Taq77] implies that with probability one,

$$
\begin{aligned}
\frac{1}{n} \sum_{i=1}^{n} G\left(X_{i}\right) & =\frac{\left(G, H_{2}\right)}{2 n} \sum_{i=1}^{n}\left(X_{i}^{2}-1\right)+o\left(n^{2 \alpha-2}\right) \quad(n \rightarrow \infty) \\
& =\frac{3\left\|\varphi \circ \Phi^{-1}\right\|_{p}^{p}}{2 n} \sum_{i=1}^{n}\left(X_{i}^{2}-1\right)+o\left(n^{2 \alpha-2}\right) .
\end{aligned}
$$

See (3.9) for the last equality. Next we start to identify the main contributions to the term $\int_{0}^{1}\left[\varphi \circ \Phi^{-1}(s)\right]^{p-1} V_{n}(s) \mathrm{d} \lambda(s)$ of the first line of (3.36). To do so, we first need to take a closer look at the Vervaat process $V_{n}$.

In accord with Csörgö and Zitikis [CsZ02, eq. 4.20],

$$
V_{n}(s)=-\frac{1}{2} \beta_{n}^{U}(s) \gamma_{n}(s)+\rho_{n}(s) .
$$

Here, $\beta_{n}^{U}$ and $\gamma_{n}$ are defined in (3.21), and

$$
\begin{aligned}
\rho_{n}(s) & :=A_{n}(s)-\frac{1}{2} B_{n}(s)+\frac{1}{2} C_{n}(s), \text { where } \\
A_{n}(s) & :=\int_{\Phi \circ \Phi_{n}^{-1}(s)}^{s}\left[\beta_{n}^{U}(u)-\beta_{n}^{U}(s)\right] \mathrm{d} \Phi^{-1}(u), \\
B_{n}(s) & :=\left[\beta_{n}^{U}(s)+\gamma_{n}^{U}(s)\right] \gamma_{n}(s), \\
\gamma_{n}^{U}(s) & :=\Phi \circ \Phi_{n}^{-1}(s)-s, \\
C_{n}(s) & :=\int_{\Phi \circ \Phi_{n}^{-1}(s)}^{s}\left[\Phi_{n}^{-1}(s)-2 \Phi^{-1}(u)+\Phi^{-1}(s)\right] \mathrm{d} u .
\end{aligned}
$$

As it turns out, the main contribution comes from $\beta_{n}^{U} \gamma_{n}$, which we rewrite as follows:

$$
\beta_{n}^{U}(s) \gamma_{n}(s)=-\frac{\left[\beta_{n}^{U}(s)\right]^{2}}{\varphi \circ \Phi^{-1}(s)}+D_{n}(s) .
$$

Here,

$$
D_{n}(s):=\beta_{n}^{U}(s)\left(\gamma_{n}(s)+\frac{\beta_{n}^{U}(s)}{\varphi \circ \Phi^{-1}(s)}\right) .
$$

We can then plug (4.5) into (4.3), and deduce the following:

$$
V_{n}(s)=\frac{\left[\beta_{n}^{U}(s)\right]^{2}}{2 \varphi \circ \Phi^{-1}(s)}+\left(\rho_{n}(s)-\frac{1}{2} D_{n}(s)\right) .
$$

Once again, it turns out that the first term of this expansion contains the main contribution to $V_{n}$. We further simplify it as follows:

$$
V_{n}(s)=\frac{\varphi \circ \Phi^{-1}(s)}{2} \bar{X}_{n}^{2}+\left(\rho_{n}(s)-\frac{1}{2} D_{n}(s)+\frac{1}{2} E_{n}(s)\right) .
$$

The new remainder term $E_{n}(s)$ is defined by the formula,

$$
E_{n}(s):=\frac{1}{\varphi \circ \Phi^{-1}(s)}\left(\left[\beta_{n}^{U}(s)\right]^{2}-\left[\varphi \circ \Phi^{-1}(s)\right]^{2} \bar{X}_{n}^{2}\right) .
$$


At this point, we can appeal to (4.8), and note that the term in (3.36) that involves the Vervaat process is nothing other than

$$
\int_{0}^{1}\left(\varphi \circ \Phi^{-1}(s)\right)^{p-1} V_{n}(s) \mathrm{d} \lambda(s)=\frac{1}{2}\left\|\varphi \circ \Phi^{-1}\right\|_{p}^{p} \bar{X}_{n}^{2}+R_{n},
$$

where

$$
R_{n}:=\int_{0}^{1}\left(\varphi \circ \Phi^{-1}(s)\right)^{p-1}\left(\rho_{n}(s)-\frac{1}{2} D_{n}(s)+\frac{1}{2} E_{n}(s)\right) \mathrm{d} \lambda(s) .
$$

The bulk of our proof of Theorem 4.1 involves proving the following claim:

$$
R_{n}=o_{\mathrm{P}}\left(n^{2 \alpha-2}\right) .
$$

For the time being, we take this for granted and complete our proof of (4.1).

By (3.36), (4.2), and (4.10), when $p \in(2, \infty)$,

$$
\begin{aligned}
& n^{2(1-\alpha)} \zeta^{\prime}(0) \\
& \quad=\frac{1}{2}\left\|\varphi \circ \Phi^{-1}\right\|_{p}\left\{\left(\frac{1}{n^{\alpha}} \sum_{i=1}^{n} X_{i}\right)^{2}+\frac{3}{n^{2 \alpha-1}} \sum_{i=1}^{n}\left(X_{i}^{2}-1\right)\right\}+o_{\mathrm{P}}(1) .
\end{aligned}
$$

Thus, Theorem 4.1 would follow from the following:

$$
\int_{0}^{1}\left(\zeta^{\prime}(\tau)-\zeta^{\prime}(0)\right) \mathrm{d} \tau=o_{\mathrm{P}}\left(n^{2 \alpha-2}\right)
$$

But this is a consequence of (3.38). To conclude, it remains to verify (4.12). A little side-calculation shows that the following five assertions suffice: Given any $\rho>1$,

$$
\left\|\left(\varphi \circ \Phi^{-1}\right)^{\rho} \Xi_{n}\right\|_{\infty}=o_{\mathrm{P}}\left(n^{2 \alpha-2}\right), \quad \text { for } \Xi=A, B, C, D, E .
$$

Henceforth, we assume that $p \in(2, \infty)$. An inspection of the following arguments reveals that when (2.6) holds for appropriate values of $\theta$ we can absorb an extra power of $\varphi \circ \Phi^{-1}$ into $\lambda$. Once more, this reduces the problem to proving (4.12) for $p>2$. This is precisely our next task.

Proof of (4.15) for $\Xi:=A$. Thanks to (3.15) and (3.21), we can write $\beta_{n}^{U}(t)=$ $r_{n} \circ \Phi^{-1}(t)-\varphi \circ \Phi^{-1}(t) \bar{X}_{n}$. Thus,

$$
\left|\beta_{n}^{U}(u)-\beta_{n}^{U}(s)\right| \leq\left\|\varphi^{\prime}\right\|_{\infty}\left|\bar{X}_{n}\right|\left|\Phi^{-1}(u)-\Phi^{-1}(s)\right|+2\left\|\gamma_{n}\right\|_{\infty} .
$$

Thanks to the monotonicity of $\Phi^{-1}$,

$$
\left|A_{n}(s)\right| \leq\left\|\varphi^{\prime}\right\|_{\infty}\left|\bar{X}_{n}\right|\left|\gamma_{n}(s)\right|^{2}+2\left\|r_{n}\right\|_{\infty}\left|\gamma_{n}(s)\right| ;
$$

cf. (3.21) for the definition of $\gamma_{n}$. Thus, when $\Xi=A$, we obtain (4.15) from (3.16), (3.17), and Proposition 3.10.

Proof of (4.15) for $\Xi:=B$. By (3.23), with probability one,

$$
\sup _{s \in(0,1)}\left|\beta_{n}^{U}(s)+\gamma_{n}^{U}(s)+\bar{X}_{n}\left[\varphi \circ \Phi^{-1}(s)-\varphi \circ \Phi_{n}^{-1}(s)\right]\right|=o\left(n^{\alpha-1}\right) .
$$

Since $\left\|\varphi^{\prime}\right\|_{\infty}<+\infty$, Taylor's expansion and (3.17) together imply that

$$
\begin{aligned}
\left|\beta_{n}^{U}(s)+\gamma_{n}^{U}(s)\right| & =O_{\mathrm{P}}\left(n^{\alpha-1}\right) \times\left|\varphi \circ \Phi^{-1}(s)-\varphi \circ \Phi_{n}^{-1}(s)\right| \\
& =O_{\mathrm{P}}\left(n^{\alpha-1}\right) \times\left|\gamma_{n}(s)\right|,
\end{aligned}
$$


where the big- $O$ terms do not depend on $s \in(0,1)$. Thus, when $\Xi=B$, we obtain (4.15) from the preceding display, used in conjunction with Proposition 3.10.

Proof of (4.15) for $\Xi:=C$. We begin by computing directly, viz.,

$$
C_{n}(s)=-\gamma_{n}(s) \gamma_{n}^{U}(s)+2 \int_{\Phi_{n}^{-1}(s)}^{\Phi^{-1}(s)}\left[\Phi^{-1}(s)-x\right] \varphi(x) \mathrm{d} x .
$$

Now it is natural to replace the $\varphi(x)$ by $\varphi \circ \Phi^{-1}(s)$ at a small cost. Namely,

$$
C_{n}(s)=-\gamma_{n}(s) \gamma_{n}^{U}(s)+\varphi \circ \Phi^{-1}(s)\left[\gamma_{n}(s)\right]^{2}+Q_{n}(s) .
$$

Here, $Q_{n}(s):=\int_{\Phi_{n}^{-1}(s)}^{\Phi^{-1}(s)}\left[\Phi^{-1}(s)-x\right]\left[\varphi(x)-\varphi \circ \Phi^{-1}(s)\right] \mathrm{d} x$, and thanks to Taylor's expansion,

$$
\left|Q_{n}(s)\right| \leq \frac{1}{3}\left\|\varphi^{\prime}\right\|_{\infty}\left|\gamma_{n}(s)\right|^{3} .
$$

On the other hand, another round of Taylor's expansion yields,

$$
\left|\gamma_{n}^{U}(s)-\varphi \circ \Phi^{-1}(s) \gamma_{n}(s)\right| \leq \frac{1}{2}\left\|\varphi^{\prime}\right\|_{\infty}\left[\gamma_{n}(s)\right]^{2} .
$$

Together, the above three displays show that $\left|C_{n}(s)\right| \leq \frac{5}{6}\left\|\varphi^{\prime}\right\|_{\infty}\left[\gamma_{n}(s)\right]^{3}$. Consequently, we obtain (4.15) with $\Xi:=C$ from this and Proposition 3.10.

Proof of (4.15) for $\Xi:=D$. We can write $\gamma_{n}^{U}(s)=\int_{\Phi^{-1}(s)}^{\Phi_{n}^{-1}(s)} \varphi(x) \mathrm{d} x$; whence,

$$
\left|\gamma_{n}^{U}(s)-\varphi \circ \Phi^{-1}(s) \gamma_{n}(s)\right| \leq\left\|\varphi^{\prime}\right\|_{\infty}\left[\gamma_{n}(s)\right]^{2} .
$$

This and (4.19) together yield the following:

$$
\left|\beta_{n}^{U}(s)+\varphi \circ \Phi^{-1}(s) \gamma_{n}(s)\right| \leq\left\|\varphi^{\prime}\right\|_{\infty}\left[\gamma_{n}(s)\right]^{2}+O_{\mathrm{P}}\left(n^{\alpha-1}\right) \times\left|\gamma_{n}(s)\right|,
$$

where the big- $O$ term does not depend on $s \in(0,1)$. By (3.22) and Proposition 3.10,

$$
\sup _{s \in(0,1)}\left[\left(\varphi \circ \Phi^{-1}(s)\right)^{p-1}\left|\frac{\beta_{n}^{U}(s)}{\varphi \circ \Phi^{-1}(s)}+\gamma_{n}(s)\right|\right]=o_{\mathrm{P}}\left(n^{\alpha-1}\right) .
$$

This and Proposition 3.10, used in conjunction, imply (4.15) for $\Xi:=D$.

Proof of (4.15) for $\Xi:=E$. Thanks to (3.15),

$$
E_{n}(s)=\left(\frac{\beta_{n}^{U}(s)}{\varphi \circ \Phi^{-1}(s)}-\bar{X}_{n}\right) r_{n} \circ \Phi^{-1}(s) .
$$

Thus, when $\Xi=E$, we obtain (4.15) from (3.16), (3.17), and (3.21).

Because of Theorem 4.1, we immediately obtain the remaining portions of Theorem 2.1 from the following:

Theorem 4.2. If $\mathcal{N}$ and $\boldsymbol{\mathcal { R }}_{\alpha}$ are independent, then for any fixed $\beta \in \mathbb{R}$,

$$
\beta^{2}\left(\frac{1}{n^{\alpha}} \sum_{j=1}^{n} X_{j}\right)^{2}+\frac{1}{n^{2 \alpha-1}} \sum_{j=1}^{n}\left(X_{j}^{2}-1\right) \stackrel{d}{\longrightarrow} \beta^{2} \mathcal{N}^{2}+\mathcal{R}_{\alpha} .
$$

Before proving this, we need a technical lemma that may be of independent interest. 
Lemma 4.3. Let $G$ denote an $n$-dimensional centered Gaussian vector with covariance matrix $\mathbf{C}=(C(i, j))_{1 \leq i, j \leq n}$. Then for any sufficiently small $|z|$,

$$
\mathrm{E}\left[e^{z \sum_{j=1}^{n} G_{i}^{2}}\right]=\exp \left(\frac{1}{2} \sum_{k=1}^{\infty} \frac{(2 z)^{k}}{k} \operatorname{Trace}\left(\mathbf{C}^{k}\right)\right) .
$$

Proof. Assume for the time being that $\operatorname{det}(\mathbf{C})>0$, and let $\mu_{1} \geq \cdots \geq \mu_{n}>0$ denote the ordered eigenvalues of $\mathbf{C}$. We then have the following easy-to-check spectral representation: $\sum_{i=1}^{n} G_{i}^{2}=\sum_{i=1}^{n} \mu_{i} W_{i}^{2}$. Here, the random variables $W_{1}, \ldots, W_{n}$ are independent and standard Gaussian. Consequently, for all $z \in \mathbb{C}$ with a sufficiently small modulus,

$$
\begin{aligned}
\mathrm{E}\left[e^{z\|G\|_{2}^{2}}\right] & =\prod_{j=1}^{n} \frac{1}{\sqrt{1-2 z \mu_{j}}}=\exp \left(-\frac{1}{2} \sum_{j=1}^{n} \log \left(1-2 z \mu_{j}\right)\right) \\
& =\exp \left(\frac{1}{2} \sum_{j=1}^{n} \sum_{k=1}^{\infty} \frac{\left(2 z \mu_{j}\right)^{k}}{k}\right) .
\end{aligned}
$$

Here, $\|\cdots\|_{2}$ denotes the usual Euclidean norm in $\mathbb{R}^{n}$, and log denotes the branch of logarithm that is real for $z>0$. The sum of the eigenvalues of a matrix equals its trace, whence the statement of Lemma 4.3 under the assumption $\operatorname{det}(\mathbf{C})>0$.

If $\operatorname{det}(\mathbf{C})=0$, then, for any fixed $\delta>0$, we first consider the random variables $G_{i, \delta}:=G_{i}+\delta Z_{i},(i=1, \ldots, n)$ where $Z_{i}$ 's are independent standard Gaussian random variables that are also independent of the $G_{i}$ 's. Then, going over the lines of the previous paragraph, we prove the desired result for the $G_{i, \delta}$ 's whose covariance matrix $\mathbf{C}+\delta^{2} \mathbf{I}$ has a strictly positive determinant. Finally, we let $\delta \downarrow 0$ and obtain the desired result.

Proof of Theorem 4.2. Let $G_{(+)}:=\left(G_{0}, G_{1}, \ldots, G_{n}\right)$, where

$$
G_{0}:=\frac{\beta}{n^{\alpha}} \sum_{j=1}^{n} X_{j} \text { and } G_{k}:=\frac{X_{k}}{n^{\alpha-\frac{1}{2}}} \text { when } k=1, \ldots, n \text {. }
$$

In this notation, statement (4.28) is rewritten as follows:

$$
\left\|G_{(+)}\right\|_{2}^{2}-n^{2(1-\alpha)} \stackrel{d}{\longrightarrow} \beta^{2} \mathcal{N}^{2}+\boldsymbol{R}_{\alpha}
$$

It is natural to aim at proving (4.32) either by using moment generating functions or characteristic functions. We shall unify both approaches by letting $z$ be a complex variable, and considering the limit, as $n \rightarrow \infty$, of

$$
\Upsilon_{n}(z):=\mathrm{E}\left[\exp \left\{z\left(\left\|G_{(+)}\right\|_{2}^{2}-n^{2(1-\alpha)}\right)\right\}\right] .
$$

To this end, we first use Lemma 4.3 to express $\Upsilon_{n}(z)$ in terms of the covariance matrix $\mathbf{C}_{(+)}$of $G_{(+)}$. We obtain the formula:

$$
\Upsilon_{n}(z)=\exp \left(\frac{1}{2} \sum_{k=1}^{\infty} \frac{(2 z)^{k}}{k} \operatorname{Trace}\left(\mathbf{C}_{(+)}^{k}\right)-z n^{2(1-\alpha)}\right) .
$$


Let $G:=\left(G_{1}, \ldots, G_{n}\right)$, and let $\mathbf{C}$ be the covariance matrix of $G$. Note that the two matrices $\mathbf{C}_{(+)}$and $\mathbf{C}$ are related by the formula

$$
\mathbf{C}_{(+)}=\left(\begin{array}{ll}
v & \eta^{\prime} \\
\eta & \mathbf{C}
\end{array}\right)
$$

with $v:=\mathrm{E}\left[G_{0}^{2}\right]$ and $\eta:=\left(\eta_{1}, \ldots, \eta_{n}\right)^{\prime}$, where $\eta_{k}:=\mathrm{E}\left[G_{0} G_{k}\right]$ for all $k=1, \ldots, n$. Consequently, $\operatorname{Trace}\left(\mathbf{C}_{(+)}\right)=v+\operatorname{Trace}(\mathbf{C})=v+n^{2(1-\alpha)}$. Thus, we can express the expectation in (4.33) in the following way:

$$
\Upsilon_{n}(z)=\exp \left(\frac{1}{2} \sum_{k=2}^{\infty} \frac{(2 z)^{k}}{k} \operatorname{Trace}\left(\mathbf{C}_{(+)}^{k}\right)+v z\right) .
$$

We shall now express $\operatorname{Trace}\left(\mathbf{C}_{(+)}^{k}\right)$ in terms of $\operatorname{Trace}\left(\mathbf{C}^{k}\right)$.

Let $C_{(+)}(i, j)$ denote the elements of $\mathbf{C}_{(+)}(1 \leq i, j \leq n+1)$. Then, for any $k \geq 2$, we have:

$$
\begin{aligned}
\operatorname{Trace}\left(\mathbf{C}_{(+)}^{k}\right) & =\sum_{j_{1}=1}^{n+1} \cdots \sum_{j_{k}=1}^{n+1} \prod_{\ell=1}^{k-1} C_{(+)}\left(j_{\ell}, j_{\ell+1}\right) \cdot C_{(+)}\left(j_{1}, j_{k}\right) \\
& =v^{k}+\operatorname{Trace}\left(\mathbf{C}^{k}\right)+\delta_{n}(k),
\end{aligned}
$$

where

$$
\delta_{n}(k):=\sum \prod_{\ell=1}^{k-1} C_{(+)}\left(j_{\ell}, j_{\ell+1}\right) \cdot C_{(+)}\left(j_{1}, j_{k}\right),
$$

and where the sum in the definition of $\delta_{n}(k)$ is computed over all $j \in\{1, \ldots, n+1\}^{k}$ such that at least one and at most $(k-1)$ of the coordinates of $j$ are equal to 1 . Then we have the first of the following two equalities:

$$
\begin{aligned}
& \Upsilon_{n}(z) \\
& =e^{\frac{1}{2} \sum_{k=2}^{\infty} k^{-1}(2 z)^{k} \operatorname{Trace}\left(\mathbf{C}^{k}\right)+\frac{1}{2} \sum_{k=1}^{\infty} k^{-1}(2 z v)^{k}+\frac{1}{2} \sum_{k=2}^{\infty} k^{-1}(2 z)^{k} \delta_{n}(k)} \\
& =\mathrm{E}\left[\exp \left(z\left(\|G\|_{2}^{2}-n^{2(1-\alpha)}\right)\right)\right] \cdot e^{\frac{1}{2} \sum_{k=1}^{\infty} k^{-1}(2 z v)^{k}+\frac{1}{2} \sum_{k=2}^{\infty} k^{-1}(2 z)^{k} \delta_{n}(k)} \\
& =\mathrm{E}\left[\exp \left(z\left(\|G\|_{2}^{2}-n^{2(1-\alpha)}\right)\right)\right] \mathrm{E}\left[e^{z G_{0}^{2}}\right] e^{\frac{1}{2} \sum_{k=2}^{\infty} k^{-1}(2 z)^{k} \delta_{n}(k)},
\end{aligned}
$$

thanks to Lemma 4.3 and the fact that $v=\mathrm{E}\left[G_{0}^{2}\right]$. We mention also that the second equality is a consequence of Lemma 4.3 and the fact that Trace $(\mathbf{C})=n^{2(1-\alpha)}$.

Now, as $n \rightarrow \infty$, the following holds boundedly:

$$
v=\mathrm{E}\left[G_{0}^{2}\right]=\frac{\beta^{2}}{n^{2 \alpha}} \mathrm{E}\left[\left(\sum_{j=1}^{n} X_{j}\right)^{2}\right] \longrightarrow \beta^{2} ;
$$

cf. (A.4). Thus, if $|z|$ is sufficiently small, then as $n$ tends to infinity,

$$
\begin{aligned}
& \Upsilon_{n}(z) \times(1+o(1)) \\
& =\mathrm{E}\left[\exp \left(z\left(\|G\|_{2}^{2}-n^{2(1-\alpha)}\right)\right)\right] \cdot \mathrm{E}\left[e^{z \beta^{2} \mathcal{N}^{2}}\right] \cdot e^{\frac{1}{2} \sum_{k=2}^{\infty} k^{-1}(2 z)^{k} \delta_{n}(k)} .
\end{aligned}
$$

Regarding the first term on the right-hand side, Rosenblatt [Ros61, pp. 434-435] has shown that as long as $|z|$ is sufficiently small, then as $n \rightarrow \infty$, it converges to 
$\mathrm{E}\left[\exp \left(z \boldsymbol{R}_{\alpha}\right)\right]$. Consequently, we obtain Theorem 4.2 if we can prove that for all sufficiently small $|z|$,

$$
\lim _{n \rightarrow \infty} \sum_{k=2}^{\infty} \frac{|2 z|^{k}}{k}\left|\delta_{n}(k)\right|=0 .
$$

To verify this, we first rewrite $\delta_{n}(k)$, for all $k \geq 2$, in the following form:

$$
\delta_{n}(k)=\sum_{m=1}^{k-1} \sum_{(m)} \prod_{\ell=1}^{k-1} C_{(+)}\left(j_{\ell}, j_{\ell+1}\right) \cdot C_{(+)}\left(j_{1}, j_{k}\right),
$$

where $\sum_{(m)}$ denotes the sum over all $j \in\{1, \ldots, n+1\}^{k}$ such that exactly $m$ of the coordinates of $j$ are equal to 1 . By symmetry, and after a relabeling of the indices, we see that these indices might as well be $j_{1}, \ldots, j_{m}$. Since there are $\left(\begin{array}{l}k \\ m\end{array}\right)$ ways to perform the relabeling, we have

$$
\delta_{n}(k)=\sum_{m=1}^{k-1}\left(\begin{array}{c}
k \\
m
\end{array}\right) \sum_{\ell=1}^{(m)} \prod_{\ell-1}^{k-1} C_{(+)}\left(j_{\ell}, j_{\ell+1}\right) \cdot C_{(+)}\left(j_{1}, j_{k}\right),
$$

where $\sum^{(m)}$ denotes the sum over all $j \in\{1, \ldots, n+1\}^{k}$ such that $j_{1}=\cdots=j_{m}=1$ and for all $\ell>m, j_{\ell} \in\{2, \ldots, n+1\}$. Now we estimate the right-hand side of (4.44) in earnest.

If we write $\varrho(k):=\operatorname{Cov}\left(X_{1}, X_{1+k}\right)$, then by (A.4), for any $\nu \in\{2, \ldots, n+1\}$,

$$
\begin{aligned}
\left|C_{(+)}(1, \nu)\right| & =\left|\operatorname{Cov}\left(G_{0}, G_{\nu-1}\right)\right|=\left|\frac{\beta}{n^{2 \alpha-\frac{1}{2}}} \sum_{j=1}^{n} \varrho(j-\nu+1)\right| \\
& =\frac{|\beta|}{n^{2 \alpha-\frac{1}{2}}}\left|1+\sum_{j<\nu-1} \varrho(j-\nu+1)+\sum_{n \geq j>\nu-1} \varrho(j-\nu+1)\right| \\
& \leq \frac{L_{1}}{n^{2 \alpha-\frac{1}{2}}}\left(1+\sum_{j<\nu-1} j^{-2(1-\alpha)}+\sum_{j>\nu-1} j^{-2(1-\alpha)}\right) \\
& \leq L_{2} n^{-\frac{1}{2}}
\end{aligned}
$$

where $L_{1}$ and $L_{2}$ depend only on $\beta$ and $\alpha \in\left(\frac{3}{4}, 1\right)$. Consequently, by (A.4),

$$
\begin{aligned}
& \left.\mid \sum_{\ell=1}^{(m)} \prod_{(+)}^{k} C_{\ell}, j_{\ell+1}\right) \cdot C_{(+)}\left(j_{1}, j_{k}\right) \mid \\
& \quad \leq \frac{L_{2}^{m+1} L_{3}^{k} n^{(k-1-m)(1-2 \alpha)} \mathfrak{S}_{k-1-m}(n)}{n^{(m+1) / 2}},
\end{aligned}
$$

where, after recalling (2.2), we have defined

$$
\mathfrak{S}_{k}(n):=\sum_{j_{1}=1}^{n} \cdots \sum_{j_{k}=1}^{n}\left[\Psi_{k}(j)\right]^{-2(1-\alpha)} .
$$

Thanks to (A.4), the constant $L_{3}$ can also be chosen to be independent of $k$ and $n$.

It is not difficult to check that there exists a constant $L_{4}$ (depends only on $\alpha$ ) such that for all $k$ and $n, \mathfrak{S}_{k}(n) \leq L_{4}^{k} n^{-k(1-2 \alpha)}$. By choosing $L_{4}$ sufficiently large, we can insure that, in addition, $\operatorname{Trace}\left(\mathbf{C}^{k}\right) \leq L_{4}^{k} n^{-k(1-2 \alpha)}$. as $n \rightarrow \infty$, uniformly 
in $k$. Thus, we have the following bound: There exists a constant $L_{5}$, that depends only on $\alpha$, such that

$$
\mathfrak{S}_{k}+\mathfrak{S}_{k}(n)+\operatorname{Trace}\left(\mathbf{C}^{k}\right) \leq L_{5}^{k} n^{-k(1-2 \alpha)} .
$$

With these observations, we now obtain the following bound that is valid for all $m \leq k-1$ :

$$
\left|\sum_{\ell=1}^{(m)} \prod_{(+)}^{k} C_{\ell}\left(j_{\ell}, j_{\ell+1}\right) \cdot C_{(+)}\left(j_{1}, j_{k}\right)\right| \leq\left(L_{2} L_{3} L_{5}\right)^{k} n^{-(m+1) / 2} .
$$

Thus, after applying (4.49) to the right-hand side of (4.44), we obtain the following:

$$
\left|\delta_{n}(k)\right| \leq\left(L_{2} L_{3} L_{5}\right)^{k} \sum_{m=1}^{k-1}\left(\begin{array}{c}
k \\
m
\end{array}\right) n^{-(m+1) / 2} \leq\left(2 L_{2} L_{4}\right)^{k} n^{-1} .
$$

Since $\alpha<1$, the above pointwise bound yields (4.42) as long as $|z|<\left(4 L_{2} L_{3} L_{5}\right)^{-1}$. The proof of Theorem 4.2 is now complete.

\section{Appendix A. Some Remarks}

Remark A.1. The Lorenz curve for $\Phi[:=$ the standard normal c.d.f. $]$ is the negative of the so-called density-quantile function, ${ }^{2}$

$$
\mathrm{GL}_{\Phi}(t)=-\varphi \circ \Phi^{-1}(t), \quad{ }^{\forall} t \in[0,1] .
$$

Indeed, after making a change of variables, we can deduce that for all $t \in[0,1]$, $\mathrm{GL}_{\Phi}(t)=\int_{-\infty}^{\Phi^{-1}(t)} x \varphi(x) \mathrm{d} x$. Equation (A.1) follows from this and the fact that $\varphi$ solves $\varphi^{\prime}(x)=-x \varphi(x)$.

Remark A.2. The function $\mathrm{GL}_{F}$ is always convex. Indeed, by the Lebesgue differentiation theorem, $F^{-1}$ has a nonnegative derivative almost everywhere. Hence, almost everywhere, $\mathrm{GL}_{F}^{\prime \prime} \geq 0$.

Remark A.3. The most natural choice of $\lambda$ is the Lebesgue measure on $[0,1]$. In this case, for any choice of $\nu \in(0,1), \int_{0}^{1} s^{-\nu}(1-s)^{-\nu} \mathrm{d} \lambda(s)<+\infty$, and we have the following simplification which can be checked by means of direct computations:

$$
\left\|\varphi \circ \Phi^{-1}\right\|_{p}^{p}=\frac{1}{(2 \pi)^{p / 2} \sqrt{p+1}}, \quad{ }^{\forall} p \geq 1 .
$$

Remark A.4. If $\left\{X_{i}\right\}_{i=1}^{\infty}$ are the increments of a fractional Brownian motion of index $\alpha \in(0,1)$, then

$$
\operatorname{Cov}\left(X_{1}, X_{1+k}\right)=\frac{(k+1)^{2 \alpha}+(k-1)^{2 \alpha}}{2}-k^{2 \alpha}, \quad{ }^{\forall} k \geq 0 .
$$

In particular,

$$
\operatorname{Cov}\left(X_{1}, X_{1+k}\right)=(\alpha+o(1))(2 \alpha-1) k^{-2(1-\alpha)}, \quad(k \rightarrow \infty) .
$$

\footnotetext{
${ }^{2}$ The function $\varphi \circ \Phi^{-1}$ is also called the Gaussian isoperimetric function in the analysis/geometry/probability literature; cf. Ros [Ros01].
} 
Another consequence of this, together with convexity, is the following well-known condition for positive/negative correlations:

$$
\operatorname{Cov}\left(X_{1}, X_{1+k}\right) \begin{cases}<0, & \text { if } \alpha \in\left(0, \frac{1}{2}\right), \\ =0, & \text { if } \alpha=\frac{1}{2}, \\ >0, & \text { if } \alpha \in\left(\frac{1}{2}, 1\right) .\end{cases}
$$

Lemma A.5. If $\left\{\omega_{k}\right\}_{k=1}^{\infty}$ is a stationary short-range dependent Gaussian process, then for any $x, y \in \mathbb{R}$,

$$
\sum_{k=1}^{\infty}\left|\operatorname{Cov}\left(\mathbf{1}_{\left\{\omega_{1} \leq x\right\}}, \mathbf{1}_{\left.\omega_{1+k} \leq y\right\}}\right)\right|<+\infty .
$$

Proof. Because $x$ and $y$ are arbitrary, we can and will assume, without loss of generality, that the $\omega_{j}$ 's are standard normal variables.

We let $c_{k}:=\operatorname{Cov}\left(\omega_{1}, \omega_{1+k}\right)$, and compute the conditional mean and variance of $\omega_{1+k}$ given $\left\{\omega_{1}=z\right\}$ to see that for any $y, z \in \mathbb{R}$, and for all integers $k \geq 1$,

$$
\mathrm{P}\left\{\omega_{1+k} \leq y \mid \omega_{1}=z\right\}=\Phi\left(\frac{y}{\sqrt{1-c_{k}^{2}}}+z c_{k}\right) \text {. }
$$

Therefore,

$$
\begin{aligned}
& \left|\operatorname{Cov}\left(\mathbf{1}_{\left\{\omega_{1} \leq x\right\}}, \mathbf{1}_{\left\{\omega_{1+k} \leq y\right\}}\right)\right| \\
& =\left|\int_{-\infty}^{x}\left[\Phi\left(\frac{y}{\sqrt{1-c_{k}^{2}}}+z c_{k}\right)-\Phi(y)\right] \mathrm{d} \Phi(z)\right| \\
& \leq \frac{1}{\sqrt{2 \pi}} \int_{-\infty}^{\infty}\left|\frac{y}{\sqrt{1-c_{k}^{2}}}-y+z c_{k}\right| \mathrm{d} \Phi(z) .
\end{aligned}
$$

In the last line we have used the fact that $\varphi$ is bounded above by $(2 \pi)^{-1 / 2}$. From this it follows easily that $\left|\operatorname{Cov}\left(\mathbf{1}_{\left\{\omega_{1} \leq x\right\}}, \mathbf{1}_{\left\{\omega_{1+k} \leq y\right\}}\right)\right| \leq \pi^{-1}\left|c_{k}\right|(1+o(1))$ as $k \rightarrow \infty$, and this completes our proof.

\section{REFERENCES}

[Bar99] J.-M. Bardet, Un test d'auto-similarité pour les processus gaussiens à accroissements stationnaires. C. R. Acad. Sci. Paris Sér. I Math. 328, 521-526, 1999.

[BaD02] G. F. Barrett and S. G. Donald, Statistical inference with generalized Gini indices of inequality and poverty, Discussion Paper 2002/01. School of Economics. Univ. of New South Wales, 2002.

[CsZ02] M. Csörgö and R. Zitikis, On the general Bahadur-Kiefer, quantile, and Vervaat processes: old and new. In: Limit Theorems in Probability and Statistics I (A Volume in Honour of Pl Rvsz. Eds.: I. Berkes, E. Csáki, M. Csörgő), 389-426. Bolyai Mathematical Society, Budapest, 2002.

[Dav68] H. A. David, Gini's mean difference rediscovered, Biometrika 55, 573-575, 1968.

[DaZ01] Y. Davydov and R. Zitikis, Generalized Lorenz curves and convexifications of stochastic processes, Publications de l'U.F.R. de Mathematiqués Pures et Appliquées, Lille 55, 1-36, 2001.

[DeT89] H. Dehling and M. S. Taqqu, The empirical process of some long-range dependent sequences with an application to U-statistics, Ann. Stat. 17, 1767-1783, 1989.

[Dob70] R. L. Dobrushin, Describing a system of random variables by conditional distributions, Th. Prob. Appl. 15, 458-486, 1970.

[Gas71] J. L. Gastwirth, A general definition of the Lorenz curve, Econometrica 39, 1037-1039, 1971. 
[Gio93] G.-M. Giorgi, A fresh look at the topical interest of the Gini concentration ratio, Metron 51, 83-98, 1993.

[Gio90] G.-M. Giorgi, Bibliographic portrait of the Gini concentration ratio, Metron 48, 183-221, 1990.

[GiS85] L. Giraitis and D. Surgailis, CLT and other limit theorems for functionals of Gaussian processes, Z. Wahrsch. Verw. Gebiete 70, 191-212, 1985.

[GuL89] X. Guyon and J. León. Convergence en loi des $H$-variations d'un processus gaussien stationnaire sur R. Ann. Inst. H. Poincaré Probab. Statist. 25, 265-282, 1989.

[Itô51] K. Itô, Multiple Wiener integral, J. Math. Soc. Japan 3, 157-164, 1951.

[Lor05] M. O. Lorenz, Methods of measuring the concentration of wealth, J. of the Amer. Stat. Assoc., 9, 209-219, 1905.

[Mar49] G. Maruyama, The harmonic analysis of stationary stochastic processes, Mem. Fac. Sci. Kyushu Univ., Ser. A 4, 45-106, 1949.

[Mos02] K. Mosler, Multivariate Dispersion, Central Regions and Depth: The Lift Zonoid Approach, Lect. Notes in Stat., Springer-Verlag, New York, 2002.

[Ros01] A. Ros, The isoperimetric problem, Lecture series at the Clay Math. Inst. Summer School on the Global Theory of Minimal Surfaces, Summer 2001, Math. Sci. Res. Inst., Berkeley, Ca. E-print available at http://www.ugr.es/ãros/isoper.htm, 2001.

[Ros61] M. Rosenblatt, Independence and dependence, Proc. 4th Berkeley Sympos. Math. Stat. and Prob., Vol. II, 431-443, Univ. California Press, Berkeley, Calif., 1961.

[ShW86] G. R. Shorack and J. A. Wellner, Empirical Processes with Applications to Statistics, Wiley, New York, 1986.

[Slep62] D. Slepian, The one-sided barrier problem for Gaussian noise, Bell System Tech. J. 42, 463-501, 1962.

[Taq75] M. S. Taqqu, Weak convergence to fractional Brownian motion and to the Rosenblatt process, Z. Wahrsch. Verw. Gebiete 31, 287-302, 1974/1975.

[Taq77] M. S. Taqqu, Law of the iterated logarithm for sums of non-linear functions of Gaussian variables that exhibit a long range dependence, Z. Wahrsch. Verw. Gebiete 40(3), 203238, 1977.

[Zit98] R. Zitikis, The Vervaat process, In: Asymptotic Methods in Probability and StatisticsA Volume in Honour of Miklós Csörgő (ed. B. Szyszkowicz), 667-694, North-Holland, Amsterdam, 1998.

[Zit02] R. Zitikis, Analysis of indices of economic inequality from a mathematical point of view, Lecture at the 11th Indonesian Mathematics Conference, State Univ. of Malang, Indonesia, Matematika 8, 772-782, 2002.

[Zit03] R. Zitikis, Asymptotic estimation of the E-Gini index, Econometric Theory, 2003 (to appear).

Université des Sciences et Technologies de Lille, Laboratoire de Statistique et Probabilités, 59655 Villeneuve d'Asce Cedex, France

E-mail address: Youri.Davydov@univ-lille1.fr

URL: http://www.univ-lille1.fr/labo-stat-proba/davydov.html

Department of Mathematics, University of Utah, 155 S. 1400 E., JWB 233, Salt Lake City, UT. 84112-0090, U.S.A.

E-mail address: davar@math.utah.edu

$U R L:$ http://www.math.utah.edu/ davar

Laboratoire de Probabilités, Université Paris Vi, F-75252 Paris Cedex 05, France

E-mail address: zhan@proba.jussieu.fr

URL: http://www.proba.jussieu.fr/pageperso/zhan

Department of Statistical and Actuarial Sciences, University of Western Ontario, London, Ontario, Canada, N6A 5B7

E-mail address: zitikis@stats.uwo.ca

$U R L:$ http://www.stats. uwo.ca/faculty/zitikis/main.htm 